RESEARCH ARTICLE

\title{
Association of Clostridioides difficile infection with specific malignant conditions
}

\author{
Chetana Vaishnavi ${ }^{1}$, Megha Sharma ${ }^{1}$, Pramod Gupta², Rakesh Kochhar ${ }^{1}$ \\ ${ }^{1}$ Department of Gastroenterology, Postgraduate Institute of Medical Education and Research, Chandigarh, India \\ ${ }^{2}$ Department of Biostatistics, Postgraduate Institute of Medical Education and Research, Chandigarh, India
}

\begin{abstract}
Objectives: Patients with concomitant diseases, particularly malignancies, are at significant risk for Clostridioides difficile infection (CDI). However, very little is known about the association between malignancy and CDI. Therefore, we evaluated the association of CDI in patients with different kinds of malignancies compared to control patients.
\end{abstract}

Methods: Patients ( $\mathrm{n}=1022)$ with specific malignancies (496 patients, 328 men), subgrouped as Adenocarcinoma (AC), Hematological malignancies (HM), Multiple myeloma (MM), Pediatric solid tumor (PST), and controls (526 controls, 325 men) without any specific diseases were enrolled in the study. Laboratory data of the patients were reviewed for demographics, antibiotic exposure, clinical symptoms, and fecal Clostridioides difficile toxin (CDT) assay.

Results: Of 1022 patients, 805 received antibiotics. CDT was positive in 168 (80.0\%) of those receiving antibiotics and in $42(20.0 \%)$ not receiving antibiotics $(p<0.001)$. Bloody diarrhea was present in $12.4 \%$ with CDT status. CDI among patients with malignancy (21.2\%) and controls (20.0\%) was insignificant $(p=0.689)$. CDT was positive in 7/25 PST (28.0\%), 24/96 AC (25.0\%), 71/332 HM (21.4\%) and 3/43 MM (7.0\%) patients, but was not significant $(p>0.05)$. Correlation of different malignant conditions with control and among themselves showed male gender in AC $(p=0.039)$ and HM ( $p=0.003)$, antibiotic exposure in MM $(p<0.001)$ and fever in PST and HM subgroups to be significant $(p<0.001)$.

Conclusion: CDT positivity was higher in males and patients exposed to antibiotics. No significant association of CDT was seen in malignant patients compared to the controls, though patients in PST and AC subgroups were more prone to CDI. J Microbiol Infect Dis 2021; 11(3):124-131.

Keywords: Clostridioides difficile infection, Hematologic malignancy, Adenocarcinoma, Multiple myeloma, Pediatric solid tumor

\section{INTRODUCTION}

In the last three decades, Clostridioides difficile infection (CDI) has become a mounting public health challenge worldwide, with a rise in the incidence in hospitalized patients. Of the several risk factors related to CDI [1], antibiotic exposure remains the foremost risk [1,2]. Among underlying conditions, malignant diseases form an essential group [3] predominantly due to prolonged hospital stay, advanced age, antibiotics, chemotherapeutic agents, and application of feeding tubes [4].
Several workers have reported that patients with solid cancers and hematologic malignancies have reduced immunity and a high risk for CDI. The gut milieu in cancer patients changes due to chemotherapy, radiotherapy, and iatrogenic processing [4]. Hospitalization rates are also reasonably high among cancer patients compared to noncancerous populations leading to increased risk for CDI.

Data in this high-risk population regarding CDI epidemiology are sparse. Though there are

Correspondence: Prof. C. Vaishnavi, ICMR Emeritus Medical Scientist, Department of Gastroenterology, PGIMER, 
several case reports of hospitalizations due to CDI associated with chemotherapy or antimicrobial use in patients with various cancers [5], only a few studies are available comparing $\mathrm{CDI}$ outbreaks among cancer patients. The first case of chemotherapyinduced CDI was reported in 1981 by Fainstein et al., [6] in a 26-year-old male with testicular embryonal cell carcinoma. This patient developed CDI with each cycle of chemotherapy but remained asymptomatic between rounds. In another study, Bilgrami et al. [7] reported 15 (7.5\%) CDI among 200 patients with various malignancies after autologous peripheral blood progenitor cell transplantation. The CDI in patients undergoing chemotherapy for gynecologic cancer was reported as $2.3-7.0 \%$, with $8.2 \%$ developing severe enterocolitis [8]. A higher risk rate for hospital-onset CDI was reported among immunocompetent cancer patients [9] and cancer patients who were immunocompromised [10] compared with noncancer patients.

As CDI is globally rising, it becomes crucial to look for the prevalence of CDI among malignant patients. In addition, timely detection of CDI patients with concomitant malignancies may be helpful for proper clinical management of the disease. The present investigation aimed to evaluate the association of $\mathrm{CDI}$ in patients with specific kinds of malignancies and compare the data with control patients.

\section{METHODS}

The study is based on retrospective analysis of fecal samples referred to the division of Microbiology of the Department of Gastroenterology for testing for C. difficile toxins (CDT) by enzyme-linked immunosorbent assay (ELISA) between October 2009 and November 2016. The Institute Ethical Committee, which operates according to the Declaration of Helsinki, cleared this project (INT/IEC/2016/2599). A total of 1059 stool samples of patients with symptoms suggestive of CDI were received and included 533 samples of patients with malignancies and 526 control patients with no inflammatory bowel disease or any other known concomitant diseases. Fecal samples were subjected to $C$. difficile toxin $A$ and/or $B$ assay using ELISA kits (DRG-International Inc, USA) with positive and negative controls. An
ELISA reader (Tecan Infinite F50, Austria) was used to read the results at $450 \mathrm{~nm}$.

Patients' details such as age, gender, underlying disease, presence, frequency and duration of diarrhea, fever, antibiotics received, other treatments were retrieved from hospital records. Patients with malignancy were separated into (i) adenocarcinoma (AC) subgroup, (ii) hematological malignancy (HM) subgroup involving leukemia and lymphoma, (iii) multiple myeloma subgroup, and (iv) solid pediatric tumor (PST)/blastoma subgroup. Thirty-seven patients who did not fit into any of the required specific subgroups of malignancies and patients with no definitive diagnosis or those with rare tumors were excluded. So finally, there were 496 patients in the malignancy group. We do not have data on the type of treatment received for malignancy by these patients. These patients were those who were admitted for diagnosis, or for starting chemotherapy, or for surgery.

\section{Statistical Analysis}

Data analysis was carried out on R-Gui Version 3.4 statistical software. Comparative analysis of the different groups was done using Chi-square, Kruskal-Wallis, and Rank sum tests. The association of CDT distribution was tested using Chi-square statistics. Similarly, the distribution of various malignancy subgroups was also analyzed and tested. When the association was significant, stratification of the data based on patients' characteristics, antibiotic received, and clinical symptoms were done for association profiling and obtained as a percent.

\section{RESULTS}

A total of 496 patients with malignancy and 526 control patients were included in the study (Table 1). Among all 1022 patients, 210 (20.5\%) tested positive for CDT; 105 for the malignancy group $(21.2 \%)$ and 105 for the control groups $(20.0 \%)$, but this difference was not found as significant $(p=0.689)$. Of the total 1022 patients, 805 had received antibiotics $(n=352$ single; $n=453$ multiple). CDT positivity was $42(20.0 \%)$ in patients who had not received any antibiotics and $168(80.0 \%)$ in those who received antibiotics. This association was highly significant $(p<0.001)$. Association between antibiotic receipt $(p=0.026)$ and fever $(p=0.004)$ in the total population was significant. 
Of the 496 patients with malignancy, 274 $(55.2 \%)$ had a fever, $286(57.7 \%)$ had watery diarrhea, and $36(7.3 \%)$ had bloody diarrhea. A total of 433 patients $(87.3 \%)$ in the malignancy group and $372(70.7 \%)$ in the control group had received antibiotics, and this association was found to be significant $(p=0.026)$. There was no difference in the occurrence of watery diarrhea or bloody diarrhea between the malignancy patients and the controls, but a significantly higher number of patients in the malignancy group had a fever $(p=0.004)$.

The CDT positivity based on the number of patients receiving antibiotics, the number of patients having fever, bloody diarrhea, and watery diarrhea are given in Table 2. The association of bloody diarrhea $(p=0.020)$ and antibiotic receipt $(p<0.001)$ with CDT status was significant. Among 496 patients with malignancy, 332 had HM, 96 had AC, 43 had $\mathrm{MM}$, and 25 had PST. Fig. 1 shows the number of CDT positivity in the controls and the different subgroups of malignancy. The number of CDT positivity differed between different malignancy subgroups. CDT was positive in 7/25 PST (28.0\%), 24/96 AC (25.0\%), 71/332 $\mathrm{HM}(21.4 \%)$ and 3/43 MM $(7.0 \%)$ patients, but was not significant ( $p>0.005)$. The CDT positivity was $21.4 \%$ in the HM subgroup, but it was not much different from the controls.
Correlation of different malignant conditions among themselves and with controls showed male gender $(p<0.001)$ and fever $(p<0.001)$ to be highly significant. On comparison with controls irrespective of CDT status, male gender was significant for $A C(p=0.039)$ and HM $(p=0.003)$, antibiotic exposure for $A C$ $(p=0.011), H M(p<0.001)$ and MM $(p=0.021)$ and fever for PST and HM $(p<0.001)$. Fig. 2 shows the percentage of CDT positivity based on presenting features and antibiotic receipt in different subgroups of malignancy.

\section{DISCUSSION}

Malignancies are an essential risk factor for CDI precipitation because of treatment with chemotherapeutic agents, which further intensify the immunosuppressive effects of neutropenia [11-13]. In the present study, CDI among patients with malignancy and controls was insignificant. CDT was positive in $7-28.0 \%$ of various types of malignancy subgroups. Correlation of different malignant conditions with control and among themselves showed male gender and fever to be highly significant. Interestingly $\mathrm{CDI}$ was found to be the least $(7.0 \%)$ associated with the MM subgroup compared to other subgroups in our study, even though antibiotic exposure was significantly associated.

Table 1. Demography and presenting features of all patients $(n=1022)$.

\begin{tabular}{lccc}
\hline Variables & Control $(\mathrm{n}=526)$ & Malignant $(\mathrm{n}=496)$ & $\mathrm{p}$-value \\
\hline Age range in years (mean $\pm \mathrm{SD})$ & $3-103(41 \pm 19)$ & $0.4-86(34.8 \pm 15.1)$ & $\mathrm{NA}$ \\
Gender (M) & $325(61.8 \%)$ & $328(66.1 \%)$ & 0.149 \\
Antibiotics exposure & $372(70.7 \%)$ & $433(87.3 \%)$ & 0.026 \\
Hospitalized & $432(82.1 \%)$ & $471(94.9 \%)$ & 0.112 \\
Fever & $212(40.3 \%)$ & $274(55.2 \%)$ & 004 \\
Watery diarrhea & $322(61.2 \%)$ & $286(57.7 \%)$ & 0.560 \\
Bloody diarrhea & $48(9.1 \%)$ & $36(7.2 \%)$ & 0.317 \\
Frequency of diarrhea (range) & $17(14-19)$ & $15(13-18)$ & $\mathrm{NA}$ \\
Duration of diarrhea (range) & $19(15-24)$ & $17(13-22.5)$ & $\mathrm{NA}$ \\
\hline
\end{tabular}




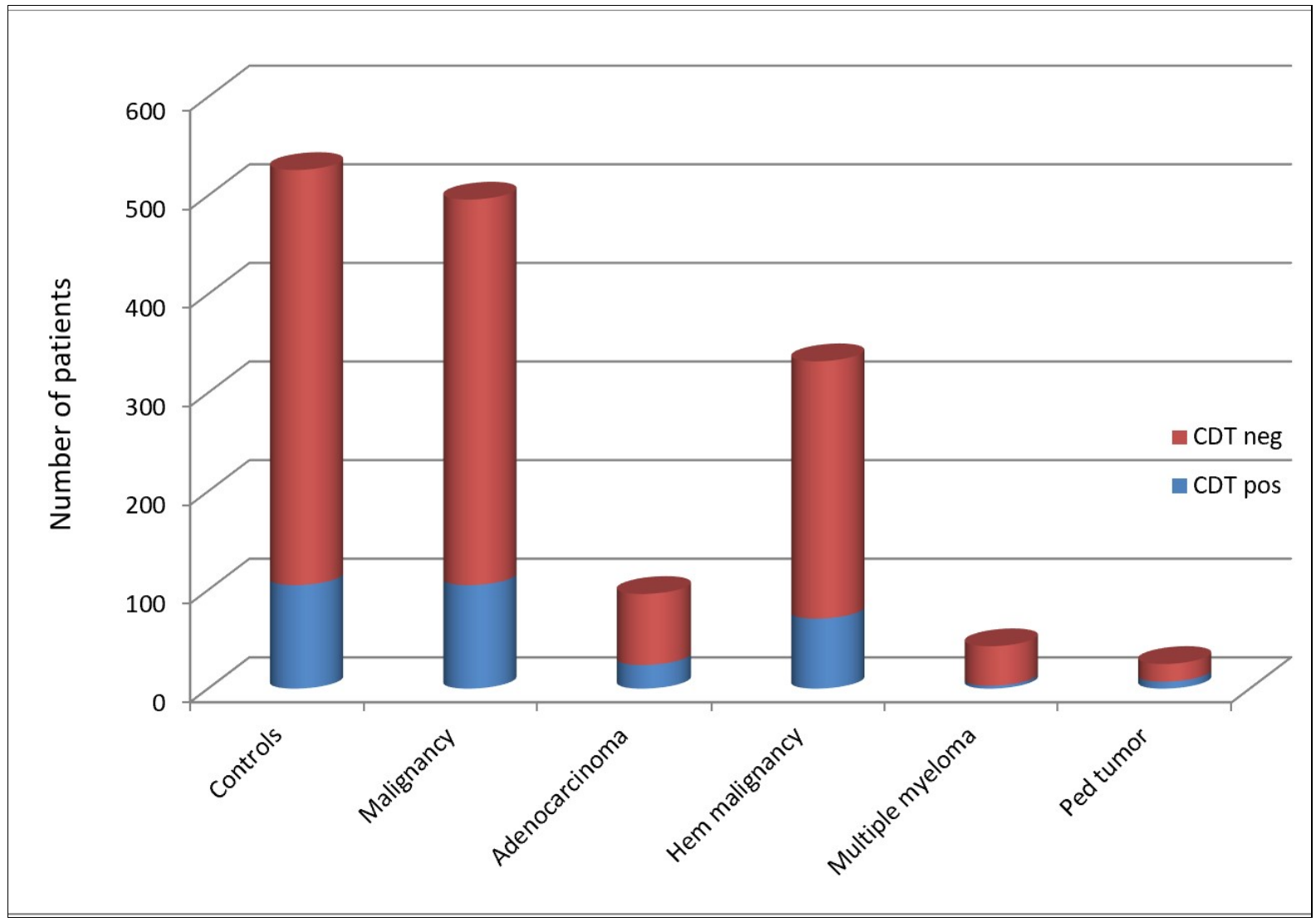

Fig. 1: Comparison of $C$. difficile toxin positivity in controls and subgroups of malignancy patients

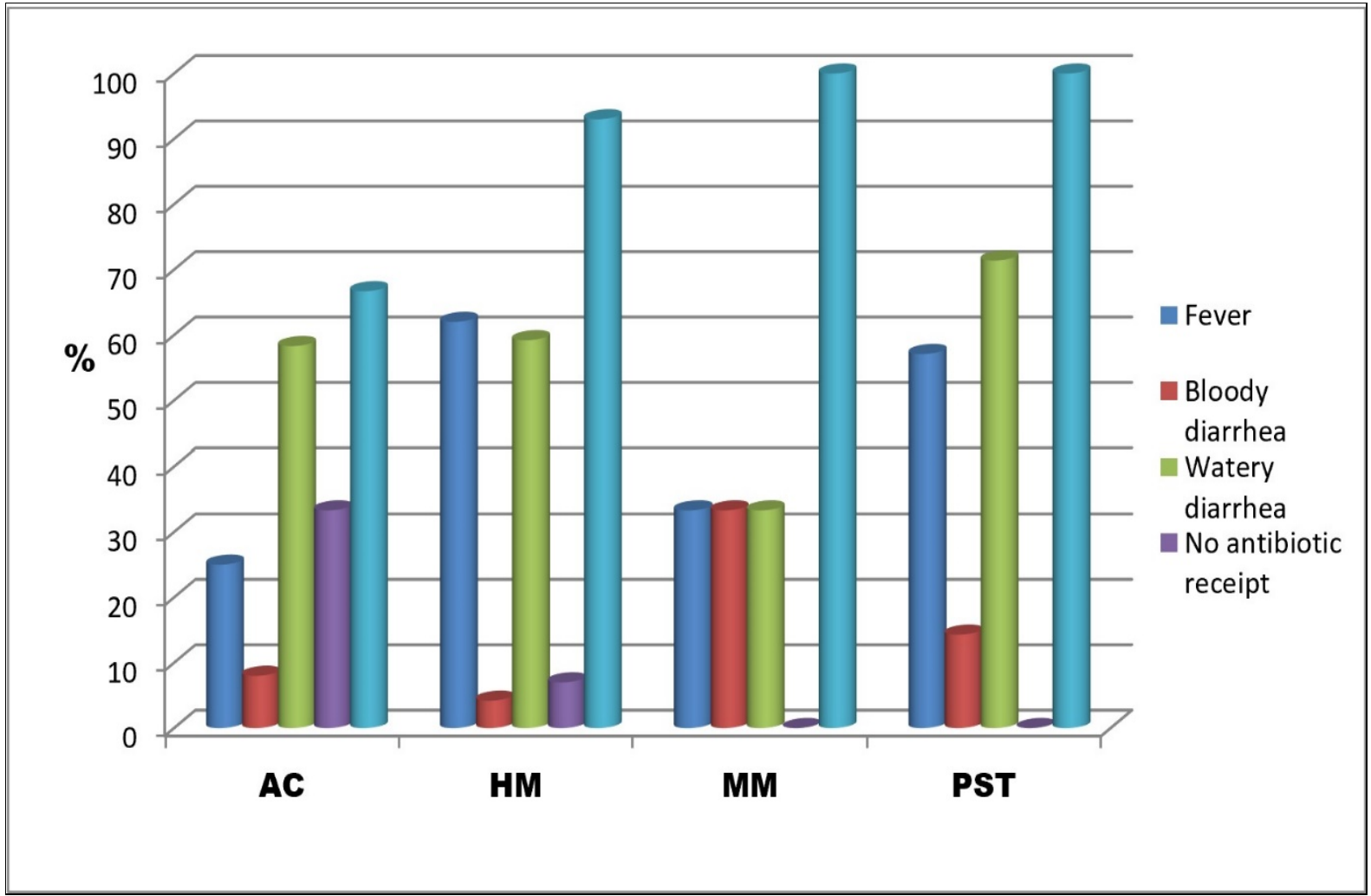

Figure 2. Presenting features and antibiotic receipt in $C$. difficile toxin positive subgroups of malignancy patients. 
Table 2. Presenting features and antibiotic receipt in CDT positive malignancy and control patients.

\begin{tabular}{|c|c|c|c|}
\hline \multirow{2}{*}{ Variables } & \multicolumn{2}{|c|}{ CDT positive $n(\%)$} & \multirow{2}{*}{$P$ value } \\
\hline & Control $(n=105)$ & Malignant $(n=105)$ & \\
\hline Fever & $42(40 \%)$ & $54(51.43 \%)$ & 0.310 \\
\hline Bloody diarrhea & $19(18.09 \%)$ & $7(6.67 \%)$ & 0.026 \\
\hline Watery diarrhea & $71(67.62 \%)$ & $62(59.05 \%)$ & 0.541 \\
\hline No antibiotic receipt & $29(27.62 \%)$ & $13(12.38 \%)$ & 0.024 \\
\hline Antibiotic receipt & $76(72.38 \%)$ & $92(87.62 \%)$ & 0.357 \\
\hline
\end{tabular}

CDT=Clostridioides difficile toxins

In the total study population of 1022 patients, 805 received single to multiple antibiotics, and among them, 168 patients were positive for CDT. This association was, however, not found to be significant. It has been reported that cytotoxic chemotherapeutic agents can precipitate $\mathrm{CDI}$ even without the use of antibiotics. Yoon et al. [11] reported 19.7\% CDI-related mortality in a study of 5594 adult patients with CDI receiving cancer treatment, which is greater than the $9.1-16.3 \%$ mortality reported by other workers [12-13]. Though chemotherapeutic agents are known to precipitate CDIs, there are no reports of CDI itself being induced by cancer. This has been further established by the increased risk of CDI in end-stage cancer patients who refuse chemotherapy [14].

In the present study, 526 controls comprised patients who had no malignancy of any kind, inflammatory bowel disease, or any other known concomitant diseases but were suspected of CDI clinically. Similarly, the malignancy group comprised of patients who had some malignancy, as mentioned earlier. We looked into the association of CDI with specific malignancy subgroups. Although CDT positivity was $25.0 \%$ among the AC subgroup, it was not significantly different from controls. However, the male gender was more affected, and antibiotics were more responsible for CDI precipitation. No literature is available in respect to the association of $\mathrm{CDI}$ with adenocarcinoma.

The CDT positivity was $21.4 \%$ in the HM subgroup, but it was different from the controls. Morris et al. [15] observed CDI in
$3(6.7 \%)$ of 45 patients with various hematologic malignancies, whereas Rampling et al. [16] reported no CDI in 20 such patients, of whom $70.0 \%$ had neutropenia. Avery et al. [17] investigated 80 patients with various malignancies after autologous peripheral blood progenitor cell transplantation and reported $\mathrm{CDI}$ in $3(3.8 \%)$ of them. Patients with neutropenia generally have hematologic malignancies and increased risk for developing CDI because of underlying diseases and treatment with a high dose of chemotherapeutic agents. It has been estimated that up to $7.0 \%$ of persons subjected to myelosuppressive chemotherapy for $\mathrm{HM}$ are likely to get $\mathrm{CDI}$, with $8.2 \%$ of them developing severe enterocolitis, compared to $2.8 \%$ incidence in the general hospitalized patient groups [18]. Panichi et al. [19] reported C. difficile in $19.0 \%$ of patients with symptomatic leukemia, with most cases also having the cytotoxin. Yoon et al. [11] reported that neutropenia independently predicted CDIrelated mortality. Sedhom et al. [20] investigated $\mathrm{CDI}$ in patients with $\mathrm{HM}$ and febrile neutropenia and concluded that such patients are likely to be at risk for a complex course of treatment in the hospital.

Patients with hematological malignancies may receive antibiotics due to a higher incidence of neutropenic fever ensuing from chemotherapy and have a longer length of hospital stay [21]. The use of ceftazidime antibiotic was an independent risk factor for CDI development in acute myeloid leukemia patients with hospitalization rates from $4.8 \%$ to $30.0 \%$ [22]. In contrast, Vehreschild et al. [23] reported carbapenems as the only independent risk 
factor in their population of acute myeloid leukemia patients. Prince et al. [24] reported that $32.0 \%$ of treated cancer patients had a longer median duration of hospital stay than non-cancer patients due to inconsistent CDI risk factors amongst patients with blood cancer. In the present study, male gender and antibiotic exposure were the factors responsible for $\mathrm{CDI}$ precipitation in the $\mathrm{HM}$ subgroup, and clinically fever was significantly associated with CDI. From a case-control study, Lee-Tsai et al. [25] reported that though the risk factors in hematological patients developing CDI did not differ from those reported from other populations, they had an increased early mortality rate, requiring new approaches for prevention and treatment.

Interestingly $\mathrm{CDI}$ was found to be the least $(7.0 \%)$ associated with the MM subgroup compared to other subgroups in our study. Apart from this, the presence of antibiotic receipt was found to be significantly associated with this subgroup. Unfortunately, no literature was available regarding the association of $\mathrm{CDI}$ with MM.

Very little literature is available regarding CDI in children with cancer. Simon et al. [26] reported a general incidence of $9.0 \%$ with a rate of 0.44 episodes $/ 1,000$ inpatient days. Apart from antimicrobial treatment, multiple hospital admissions and the presence of acute leukemia or lymphoma but not solid tumors are also identified risk factors for CDI development. Castagnola et al. [27] reported that all the drugs administered to children with cancer to treat infectious complications were related to CDI development. However, CDI was found in patients with solid tumors and not in those treated for acute leukemia, lymphoma, or in those receiving hemopoietic stem cell transplants. This was probably due to the administration of specific drugs to patients with solid tumors or because of the involvement of neutrophils in the pathophysiology of CDI. Garzotto et al. [28], in a small cohort, observed no relationship between specific types of antineoplastic therapy and CDI or between a solid tumor type and CDI.

In the present study, we found a high association of CDT positivity $(28.0 \%)$ in the subgroup of patients with PST/blastoma. However, it was non-significant compared to the control group. In addition, fever was found to be a significant clinical symptom in this subgroup. Patients with localized tumors receive radiotherapy alone or a moderate simultaneous dose of chemotherapy leading to higher confined toxicity. Apart from concomitant chemotherapy, the patients generally also receive antibiotics, leading to higher precipitation of $\mathrm{CDI}$.

Kamthan et al. [29] suggested that patients with severe bloody diarrhea who receive chemotherapy should be immediately tested for CDI even without any history of antibiotic receipt. However, in our study, bloody diarrhea was present in only $12.4 \%$ of patients positive for CDT and, therefore, not an essential feature of $\mathrm{CDI}$.

There are some limitations to our study. The main limitation of this study is its retrospective nature. Thus, we had no access to any patients' prior treatment, including chemotherapy, radiotherapy, and surgery details. Even though we found a higher association of CDI in patients with PST/ blastomas and $\mathrm{AC}$, the association was not statistically significant because of the smaller sample size in the subgroups studied. The increased $\mathrm{CDI}$ association in these subgroups could be because of $\mathrm{C}$. difficile exposure due to recurrent or more extended hospitalizations, suppression of immunity with the disease or drug treatment, exposure to medications, and similar reasons that alter the gut microbiota. In the present study, receipt of antibiotics was an essential factor in patients with CDI. We did not find a significant association of $\mathrm{CDI}$ in malignant patients when compared to the control patients as reported by others as the hypervirulent strain (NAP1/BI/027) is not present in our population [30]. The strength of the present study is that the association of CDI with different kinds of malignancies has been studied for the first time. However, prospective studies should be conducted to collect more information about this with larger sample size.

Rapid diagnosis and proper CDI management are essential in patients with malignancies as a response to $\mathrm{CDI}$ treatment are considerably lower among patients with cancer than those without cancer. In addition, the severity of $\mathrm{CDI}$ in patients with hematologic malignancies may affect the choice of treatment. However, assessing the severity of $\mathrm{CDI}$ in cancer patients can be complicated because neutropenia is a side effect of routine 
chemotherapy regimens and may complicate the choice of antibiotics.

In conclusion, CDI was not significantly associated with malignancy in our setup, though CDT was more common in patients in the PST and the AC subgroups. In addition, CDT positivity was highly associated with the male gender and in patients exposed to antibiotics.

\section{ACKNOWLEDGMENTS}

The Indian Council of Medical Research is gratefully acknowledged for the post of Emeritus Medical Scientist given to CV.

Declaration of conflicting interest: The author(s) declare no potential conflicts of interest concerning this article's research, authorship, and/or publication.

Financial disclosure: No financial support was received for this study

\section{REFERENCES}

1. Vaishnavi C. Established and potential risk factors for Clostridium difficile infection. Indian J. Med. Microbiol 2009;27: 291-302.

2. Kim SC, Seo MY, Lee JY, et al. Advanced chronic kidney disease: a strong risk factor for Clostridium difficile infection. Korean $\mathrm{J}$ Intern Med 2016; 31:125-133.

3. Vaishnavi C, Gupta PK, Sharma M, Kochhar R. Pancreatic disease patients are at higher risk for Clostridium difficile infection compared to those with other co-morbidities. Gut Pathogens 2019; 11: Article number: 17.

4. McCaleb RV, Gandhi AS, Clark SM, Clemmons $A B$. Clinical outcomes of acid suppressive therapy use in hematology/oncology patients at an academic medical center. Ann Pharmacother 2016; 50:541-547.

5. Yang SW, Moon W. A case of pseudomembranous colitis after paclitaxel and carboplatin chemotherapy. Korean J Gastroenterol2009; 54:328-332.

6. Fainstein V, Bodey GP, Fekety R. Relapsing pseudomembranous colitis associated with cancer chemotherapy. J Infect Dis 1981; 143:865.

7. Bilgrami S, Feingold JM, Dorsky D, et al. Incidence and outcome of Clostridium difficile infection following autologous peripheral blood stem cell transplantation. Bone Marrow Transplant 1999; 23:1039-1042.

8. Husain A, Aptaker L, Spriggs DR, Barakat RR. Gastrointestinal toxicity and Clostridium difficile diarrhoea in patients treated with paclitaxel containing chemotherapy regimens. Gynecol Oncol 1998; 71:104-107.

9. Chopra T, Chandrasekar P, Salimnia $H$, et al. Recent epidemiology of Clostridium difficile infection during hematopoietic stem cell transplantation. Clin Transpl 2011; 25: E82-E87.

10. Delgado A, Reveles IA, Cabello FT, Reveles KR. Poorer outcomes among cancer patients diagnosed with Clostridium difficile infections in United States community hospitals. BMC Infectious Dis 2017; 17:448.

11. Yoon YK, Kim MJ, Sohn JW, et al. Predictors of mortality attributable to Clostridium difficile infection in patients with underlying malignancy. Support Care Cancer 2014; 22:2039-2048.

12. Apostolopoulou E, Raftopoulos V, Terzis K, Elefsiniotis I. Infection probability score: a predictor of Clostridium difficile-associated disease onset in patients with haematological malignancy. Eur J Oncol Nurs 2011; 15:404-409.

13. Wang MS, Evans CT, Rodriguez T, Gerding DN, Johnson S. Clostridium difficile infection and limitations of markers for severity in patients with hematologic malignancy. Infect Control Hosp Epidemiol 2013; 34:127-132.

14. Kyne L, Sougioultzis S, McFarland LV, Kelly CP. Underlying disease severity as a major risk factor for nosocomial Clostridium difficile diarrhoea. Infect Control Hosp Epidemiol 2002; 23:653-659.

15. Morris JG Jr, Jarvis WR, Nunez-Montiel OL, et al. Clostridium difficile. Colonization and toxin production in a cohort of patients with malignant hematologic disorders. Arch Intern Med 1984; 144:967-969.

16. Rampling A, Warren RE, Bevan PC, et al. Clostridium difficile in haematological malignancy; $\mathrm{J}$ Clin Pathol 1985; 38:445-451.

17. Avery R, Pohlman B, Adal K, et al. High prevalence of diarrhea but infrequency of documented Clostridium difficile in autologous peripheral blood progenitor cell transplant recipients. Bone Marrow Transplantation 2000; 25:67-69.

18. Loo VG, Bourgault AM, Poirier L, et al. Host and pathogen factors for Clostridium difficile infection and colonization. N Engl J Med 2011; 365:16931703.

19. Panichi G, Pantosti A, Gentile G, et al. Clostridium difficile colitis in leukemia patients. Radiation Oncol 2011; 6:89.

20. Sedhom D, Elsaid M, Sedhom R. Clostridium difficile infection in patients with hematologic malignancy and neutropenic fever: A clinical overview. Am J Gastroenterol 2016; Abstract 149, page $\mathrm{s} 83$.

21. Stewart DB, Yacoub E, Zhu J. Chemotherapy patients with $\mathrm{C}$. difficile colitis have outcomes similar to immunocompetent $\mathrm{C}$. difficile patients. J Gastrointest Surg 2012; 16:1566-1572.

22. Schalk E, Bohr UR, Konig B, Scheinpflug K, Mohren M. Clostridium difficile-associated diarrhoea, a frequent complication in patients with acute myeloid leukaemia. Ann Hematol 2010; 89:914. 
23. Vehreschild MJGT, Weitershagen D, Biehl LM, et al. Biol Blood Marrow Transplant 2014; 20: $823 e 828$.

24. Prince RM, Krzyzanowska MK, Atenafu EG. Frequency and predictors of hospitalization during chemotherapy: a systematic review. J Clin Oncol 2015;33: Suppl; Abstr 6583.

25. Lee-Tsai YL, Luna-Santiago R, DemichelisGómez R, et al. Determining the risk factors associated with the development of Clostridium difficile infection in patients with hematological diseases. Blood Res 2019; 54:120-124.

26. Simon A, Ammann RA, Bode $U$, et al. Healthcare-associated infections in pediatric cancer patients: results of a prospective surveillance study from university hospitals in Germany and Switzerland. BMC Infect Dis 2008; 8:70

27. Castagnola E, Battaglia T, Bandettini R, et al. Clostridium difficile-associated disease in children with solid tumors. Support Care Cancer 2009; 17:321-324.

28. Rodrıguez GA, M'erida GA, Muñoz UN, et al. Risk factors associated with Clostridium difficile infection in adult oncology patients. Support Care Cancer 2015; 23:1569-1577.

29. Kamthan AG, Bruckner HW, Hirschman SZ. Agus SG. Clostridium difficile diarrhoea induced by cancer chemotherapy. Arch Intern Med 1992; 152:1715-1717.

30. Singh M, Vaishnavi C, Kochhar R, Mahmood S. Toxigenic Clostridium difficile isolates from clinically significant diarrhoea in patients from a tertiary care centre. Indian J Med Res. 2017; 145:840-846. 